\title{
CCD X-ray detectors: opportunities and challenges
}

\author{
Roy Clarke \\ Harrison Randall Laboratory of Physics, University of Michigan, Ann Arbor, MI 48109-1120, USA
}

\begin{abstract}
Charge coupled device (CCD) arrays are showing considerable promise for a variety of X-ray imaging applications. Performance characteristics such as high dynamic range, versatile readout and data-storage modes, and time-resolved capabilities, present interesting opportunities for new kinds of experiments on high-brilliance sources. We illustrate some of these possibilities with results of our recent synchrotron radiation studies using CCDs as high-resolution position-sensitive detectors. While important hurdles have already been overcome, several challenges remain for the effective exploitation of CCDs in X-ray applications. Among these are requirements for further increases in spatial resolution, radiation hardening, and very-high-speed data acquisition. Ongoing developments and future directions are discussed in this context.
\end{abstract}

\section{Introduction}

Charge coupled devices (CCDs) are currently the pre-eminent choice for a wide range of optical imaging applications [1], ranging from video cameras to highsensitivity imagers for astronomical telescopes. In recent years the utility of CCDs as X-ray detectors has gained increasing recognition and this technology is emerging as a major approach to new generations of $\mathrm{X}$-ray detectors for synchrotron radiation research.

With the advent of high-brilliance synchrotron sources such as ESRF and APS, X-ray detectors will need to perform at a far more demanding level than at present. They must be able to handle large fluxes (i.e. have large dynamic range), have inherently fast data handling capabilities and be capable of imaging with high spatial resolution. Some of these characteristics compete with each other so that it is hard to meet a wide range of requirements with a single detector design. However, CCD arrays are very versatile and lend themselves well to different detector performance requirements.

In this paper we summarize some of the current directions in CCD X-ray detector development in the context of synchrotron radiation research. We will illustrate the available opportunities with some of our recent results, pointing out where additional work is required to meet the challenges of third-generation synchrotron sources.

\section{Experimental requirements}

In general, synchrotron radiation $X$-ray experiments require three main components: a bright tunable source, efficient detector systems and well-designed $\mathrm{X}$-ray optics. These three requirements are clearly interrelated and are each equally important. A good example is the demanding set of experiments recently carried out by our group at beamline $\mathrm{X}-16 \mathrm{~B}$ at the National Synchrotron Light Source, NSLS, to demonstrate the feasibility of performing fast time-resolved $\mathrm{X}$-ray scattering measurements.

The experimental layout for these experiments is shown in Fig. 1: a Lemonnier-type [2] asymmetric-cut Ge (111) bent crystal monochromatizes the white beam providing narrow-band $\left(\Delta E / E \approx 2 \times 10^{-4}\right)$ radiation which is angularly dispersed in the horizontal plane. $\Delta \theta \approx 200 \operatorname{arcsec}$ (see Fig. 2). For the time-resolved $\mathrm{X}$-ray scattering described here, the horizontal angular dispersion provides a fan of nearly monochromatic $\mathrm{X}$ rays incident on the sample over a range of Bragg angles. When used in conjunction with a position sensitive detector, such as a CCD pixel array, this angulardispersive method is capable of recording X-ray scattering intensities simultaneously over small portions of reciprocal space close to a Bragg peak (Fig. 3). This is accomplished without mechanically scanning either the crystal or the detector. The method is particularly well suited to measuring time-dependent strain profiles and surface/interface structures in samples which are close to the ideal crystal limit, that is to say structures with rocking curves narrower than a few hundred arcseconds [3]. This situation includes some very important classes of technological materials including semiconductor heterostructures, magnetic multilayers, and superconducting thin films.

\section{CCD imaging and readout}

The basic operation of a CCD array can be readily understood with reference to Fig. 4. Each pixel in the detector array acts as an MOS-type capacitor storing 


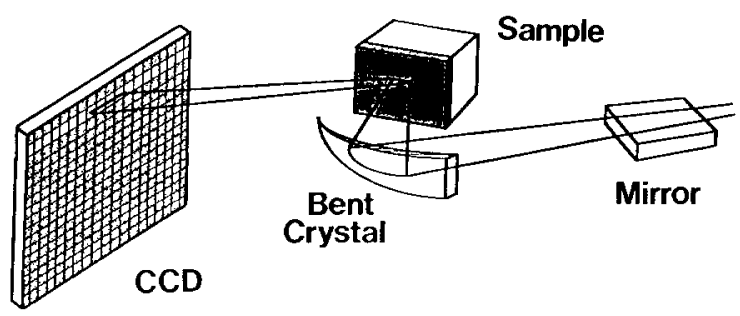

Fig. 1. Experımental arrangement for angular dispersive realtıme X-ray scattering studies using a CCD detector. The bent crystal is asymmetric cut $\mathrm{Ge}(111)$ The X-ray optics correspond to the configuration of the X-16B AT\&T Bell Labs beam line at the National Synchrotron Light Source.

photo-generated charge under a polysilicon gate electrode. 1 electron per $3.65 \mathrm{eV}$ of incident photon energy is generated and the full-well depth is typically $3-5 \times$ $10^{5}$ electrons for a $20 \mu \mathrm{m} \times 20 \mu \mathrm{m}$ pixel. The full-well depth is proportional to the pixel area.

During readout the charge accumulated in each pixel is transferred row-by-row to the serial (output) register by a sequence of clocking pulses applied to the parallel register. Once it reaches the serial register a row of pixels is read out pixel-by-pixel through an on-chip output amplifier. After further amplification the analog output can be digitized and stored in memory. To reduce readout noise associated with the output amplifier reset function, correlated double sampling [4] (CDS) is frequently used. CDS can be implemented on the chip in some of the newer designs, or it can be performed with an external circuit.

Typically CCD chips used in scientific applications are cooled to reduce dark current generation. Typical numbers at ambient temperature range from a few hundred to a few thousand electrons per pixel per second, reducing by a factor of 2 for every $\sim 8^{\circ} \mathrm{C}$ drop in temperature. Thus, for most applications, except for very long exposure times, thermoelectric cooling to

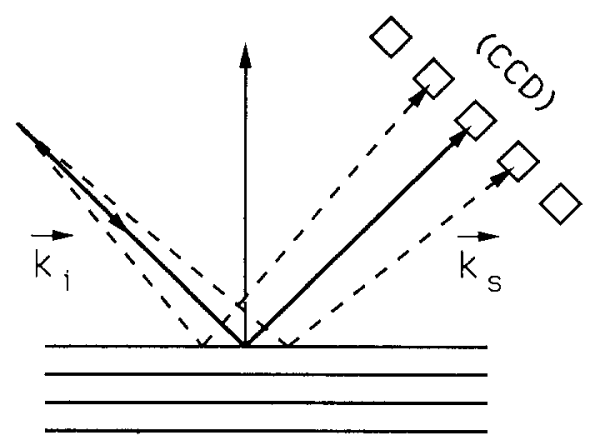

Fig. 3. Angular dispersive scattering geometry using a CCD array detector.

approximately $-30^{\circ} \mathrm{C}$ is sufficient. For some of the very fast experiments referred to below, ambient temperature operation will be satisfactory.

For more details on the operation of scientific CCDs [5], their development history [6], and specific application to X-ray detection $[7,8]$, the reader is directed to a number of excellent reviews in the literature.

\section{Direct detection of $X$ rays}

Several X-ray detectors based on CCDs employ phosphors or scintillators to convert the $\mathrm{X}$ rays to visible photons. Frequently, fiber optic tapers are used as face plates in such detectors. For our specific applications, requiring very fast readout and moderately high spatial resolution, the use of such converters can be detrimental. Accordingly, we have focused most of our CCD work on direct detection of $\mathrm{X}$ rays [9].

Direct detection is made possible by the approximate matching of the absorption depth of silicon $(\sim 100$ $\mu \mathrm{m}$ for $8 \mathrm{keV} \mathrm{X}$ rays) with the MOS-depletion-layer depth, which depends mainly on the dopant concentra-
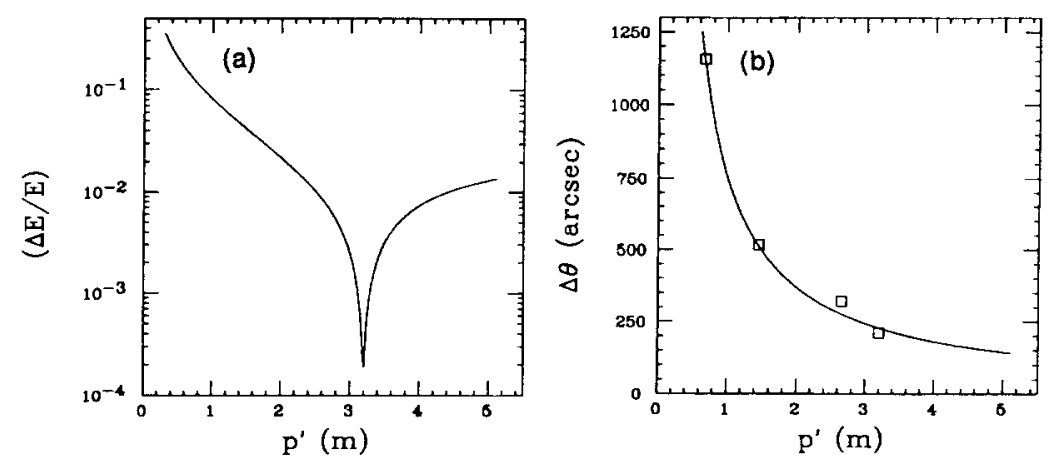

Fig. 2 Calculated dependence of (a) energy bandpass and (b) angular divergence of synchrotron beam on the monochromator-focus distance, $p^{\prime}$. The data points in (b) were measured on beam line X-16B at the NSLS. 


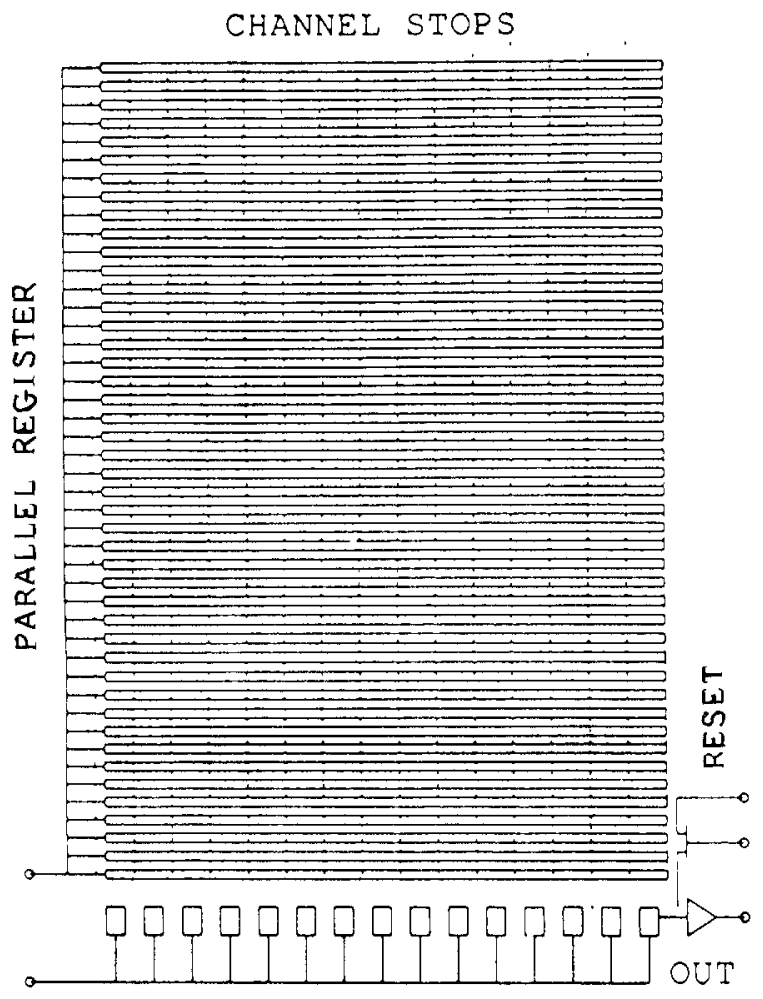

SERIAL REGISTER

Fig. 4. Schematic layout of a single-output CCD chip.

tion. For most commercially-available CCDs the depletion depths are fairly small (4-15 $\mu \mathrm{m}$, typically). Nevertheless, such devices offer useful detector quantum efficiencies (DQEs) in the range $5-15 \%$ for $X$ rays in the $10-20 \mathrm{keV}$ range. With current manufacturing techniques it is possible to produce depletion layers as thick as $50 \mu \mathrm{m}$ using very low dopant concentrations $\left(<10^{12} \mathrm{~cm}^{-3}\right)$. Such "deep depletion" devices have $\mathrm{X}$-ray DQEs approaching $100 \%$, albeit at the expense of decreased spatial resolution.

Most CCD chips are intended for use in front-side illumination. For $\mathrm{X}$-ray photon energies in the range $<10 \mathrm{keV}$ it can be advantageous to illuminate the detector from the back so that absorption by the gate oxide and electrode structure is avoided. In this mode the substrate of the device is mechanically thinned (to $\sim 10 \mu \mathrm{m}$ ). Several successful back-thinned devices have been reported and the process is now considered to be routine. For our purposes in the X-ray region, either back- or front-side illumination can be used. It remains to be seen which approach will be more favorable for detector applications.

\section{Radiation damage}

All silicon devices that are used for direct X-ray detection suffer to some degree from radiation damage. An important question is whether such damage is acceptable in terms of useful operation of the detector. First, we describe briefly what kind of effects are produced in the energy range of interest.

The radiation damage for $\mathrm{X}$ rays in the $5-20 \mathrm{keV}$ region manifests itself as traps in the oxide layer. The main effect is to produce an increase in the dark current (see Fig. 5). However, from this data [10] we see that up to $0.5 \mathrm{Mrad}$ dose has to be absorbed before the additional dark current exceeds the signal from one $\mathrm{X}$-ray photon at $10 \mathrm{keV}$. For practical purposes, then, radiation damage has a minimal effect on a CCD detector used in this mode. The effects of this kind of radiation damage (as opposed to the lattice damage created by energetic particles and gamma-rays) can be reversed by UV illumination or by a simple thermal annealing process [11]. We have not observed any significant changes in the charge transfer efficiency (CTE) for the CCDs we have evaluated.

Our conclusion is that radiation damage for 5-20 $\mathrm{keV} \mathrm{X}$ rays is not a serious limitation for all but the most demanding applications (e.g., very low photon flux requiring long exposures). We have operated a detector based on a Loral MPP CCD for over $200 \mathrm{~h}$ in diffuse scattering experiments on an NSLS bendingmagnet beam line. This exposure produced no noticeable degradation of the CCD chip at the level of 12-bit resolution.

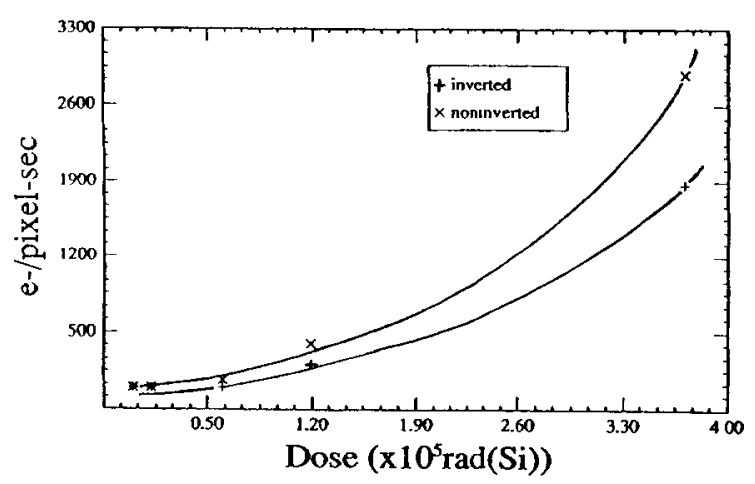

Fig. 5. Excess dark current generated by X-ray radiation damage in Loral MPP CCD. The "inverted" mode refers to an operating condition in which one or more of the array clocks are negatively biased sufficient to pin the $\mathrm{Si}-\mathrm{SiO}_{2}$ interface potential to the substrate potential. This reduces overall dark current but also decreases the full well capacity. Data taken on a rotating anode source with $8 \mathrm{keV} \mathrm{X}$ rays [10]. 


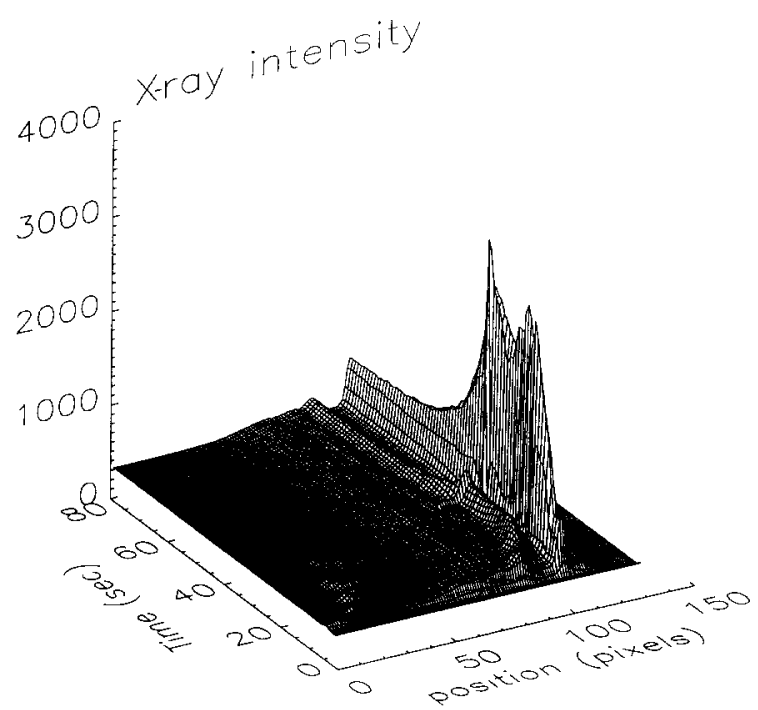

Fig. 6. CCD used in "streak mode" to record time-resolved $\mathrm{X}$-ray scattering data on the kinetics of annealing of epitaxial $\mathrm{Ge}$ on GaAs. Time (increasing temperature) is to the left. The large ridge of intensity at around 115 pixels corresponds to the substrate and the smaller ridge in front of it corresponds to the Ge overlayer. The splitting between the two peaks is approximately 300 arcsec at the commencement of annealing. The sample was grown at a substrate temperature of $350^{\circ} \mathrm{C}$ and the maximum annealing temperature was $550^{\circ} \mathrm{C}$.

One reason for the relative radiation hardness of the MPP [12] chip relates to the use of shallow ion-implants in the gate electrode structure which "pin" the subelectrode potentials and help to stabilize the charge transfer process. Our earlier experiments [13] performed with a "virtual phase" CCD (TI 4849) [14] showed that this deeply-implanted device had an even greater tolerance to radiation damage. However, the deep implants in that case have detrimental side effects; for example, the CTE was inherently poorer than that of the MPP design.

\section{Results using direct X-ray detection}

Here we illustrate the usefulness of direct detection of $\mathrm{X}$ rays by $\mathrm{CCD}$ s with an example drawn from our recent studies on beam line X16-B at NSLS. This example emphasizes both the high spatial resolution inherent in CCDs as well as the potential for very fast time-resolved operation.

In Fig. 6 we show a readout from a Loral $516 \times 516$ $\mathrm{CCD}$ which has been used at a $1 \mathrm{MHz}$ readout rate to record in real time the diffraction pattern from an epitaxial layer of Ge grown on GaAs [15]. This is particularly challenging because $\mathrm{Ge}$ and GaAs have very close lattice matching $(\sim 0.2 \%)$ and the epilayer grows pseudomorphically. Therefore, it is very difficult to image the $\mathrm{Ge}$ and GaAs peaks separately with precision using standard diffraction techniques. The experimental arrangement is shown in Fig. 1.

In order to obtain real-time information the one-dimensional diffraction pattern is imaged on a single row of the CCD. Utilizing the parallel transfer capability of the device we can record changes in the diffraction pattern as a function of time as the pattern is electronically "streaked" downwards towards the serial output register. In this case the temperature of the sample was ramped in a rapid thermal annealing cycle to investigate the kinetics of structural changes at the epitaxial interface. The data in Fig. 6 illustrate that we could track changes in lattice parameter with a precision of $\sim 1$ in $10^{6}$ on a time scale of $\sim 1 \mathrm{~ms}$. The temporal resolution in this experiment was limited by photon flux, not by the speed of the detector. Row-transfer times down to a few hundred nanoseconds are feasible in some types of CCD arrays, allowing measurements to be made within a single bunch period $(\sim 3 \mu$ s at APS).

In the above example, the data were acquired using a custom-made VME buffer [16] and transferred to hard disc through a SCUSI (Small Computer System Interface). The data is displayed on a SUN workstation, which also handles global control of the detector system.

\section{Future prospects and challenges}

The time-resolved techniques described here are the result of matching efficient X-ray optics to fast parallel data acquisition methods based on CCDs. We can expect further dramatic increases in readout speed as multiple output devices are brought into operation. These are CCDs in which the active area is divided into segments, each having its own output amplifier and CDS circuitry. Fast-framing devices are now available with 16 or 32 output channels [17]. The compatibility of some multiport devices with direct X-ray detection is currently under evaluation by our group.

One of the pressing challenges for CCD X-ray detectors obviously relates to very-high-speed data acquisition and analysis. Fortunately the technology in this area continues to enjoy an exponential evolution, and it seems likely that the speed and capacity of $A / D$ converters, CPU, computer buses, mass memory, etc., will keep pace with the detector community's needs. For example, our group is working on designs of parallel detector systems which will operate at rates in 
excess of $1 \mathrm{Gbits}^{-1}$ by means of current technology. While it may be feasible to acquire data at such high rates, it will clearly require some thought as to how to access the results in an efficient and useful way. Thus, software development for visualization and graphical user interfaces will be extremely important. The development of smart pixel devices [18], which are selective as to the access of useful information, will be a significant improvement.

We have not discussed developments which are underway towards increasing the active area of CCD detectors. In some fields such as protein crystallography, and medical imaging, this is an important requirement. This challenge is being met by two different approaches, one using an array of fiber-optic-coupled CCDs [19] and the other using large-area image intensifiers [20].

Another important challenge is concerned with achieving still higher spatial resolution. Currently the CCD with the smallest pixel size is the Kodak KAF1400 chip with $6.8 \times 6.8 \mu \mathrm{m}$ pixels. Since there are practical considerations which limit the minimum pixel size, optical demagnification schemes will be needed to further improve spatial resolution. An important requirement for high resolution work arises from experiments on coherent X-ray beams, e.g. speckle interferometry [21] and photon correlation spectroscopy [22]. Coherent X-ray studies, made possible by the extreme brilliance of undulator sources on the third generation synchrotrons, present some of the most demanding needs for the detector systems now under development.

In summary, CCD-array technology offers interesting approaches to many of the detector needs for synchrotron radiation. It is quite clear that the unique capabilities of the new generation of sources that are coming into use over the next few years will not be fully exploited without appropriate detector development.

\section{Acknowledgments}

Some of the work described above was carried out in collaboration with W.P. Lowe, R. MacHarrie, S. Dierker and R. Pindak under the University of Michigan/Howard University/ AT \& T Bell Labs Collaborative Access Team (MHATT-CAT) at the Advanced Photon Source. We also acknowledge collaboration with Brian Rodricks at the Advanced Photon Source.
Funding for this work was provided by NSF Grant DMR-9214220 and DoE Grant DE-FG05-91ER79042.

\section{References}

[1] For a general overview, see: Photoelectronic Image Devices (The McGee Symposium), ed. B.L. Morgan, Institute of Physics Conf. Ser. No. 121 (IOP, NY, 1991).

[2] M. Lemonnier, R. Fourme, F. Rousseaux and R. Kahn, Nucl. Instr. and Meth. 152 (1978) 173.

[3] R. Clarke, W. Dos Passos, Y.J. Chan, D. Pavlidis, W. Lowe, B. Rodricks and C. Brizard, in: Physical Concepts of Materials for Novel Optoelectronic Device Applications, SPIE 1361 (1990) 2.

[4] G.R. Hopkinson and D.H. Lumb, J. Phys. E. 15 (1982) 1214.

[5] J. Janesick, T. Elliott, S. Collins, M. Blouke and J. Freeman, Opt. Eng. 26 (1987) 692.

[6] J. Janesick and T. Elliott in: Astronomical Society of Pacific Conf. Ser. '91.

[7] D.J. Burt, Proc. ESA Symp. on Photon Detectors for Space Instrumentation (ESA-SP-356, 1992) p. 57.

[8] N.M. Allinson, R. Brammer, J.R. Helliwell, S. Harrop, B.G. Magorrian and T. Wan, J. X-ray Sci. Technol. 1 (1989) 143.

[9] R. Clarke, Nucl. Instr. and Meth. A 291 (1990) 117; W. Lowe, R. Clarke, W. Dos Passos, B. Rodricks and C. Brizard, Rev. Sci Instr. 63 (1992) 704.

[10] R. MacHarrie, G. Sims and W.P. Lowe, to be published.

[11] B.G. Magorrian and N.M. Allinson, Nucl. Instr. and Meth. A 273 (1988) 599.

[12] J. Janesick, T. Elliott, M. Blouke and B. Corrie, in: Optical Sensors and Electronic Photography, eds. M. Blouke and D. Pophal, Proc. SPIE 1071 (1989) 153.

[13] B. Rodricks, R. Clarke, R. Smithers and A. Fontaine, Rev. Sci. Instr. 60 (1989) 2586.

[14] Texas Instruments CCD, no longer manufactured.

[15] R. Clarke, D. Barlett, R. MacHarrie and C. Taylor, to be published.

[16] Manufactured by Photometrics Inc.

[17] For example, the fast framing devices manufactured by David Sarnoff Research Center, Princeton, NJ.

[18] S. Gruner, private communication.

[19] I. Naday, S. Ross, M. Kanyo, E. Westbrook and M. Westbrook, these Proceedings (8th Nat. Conf. on Synchrotron Radiation Instrumentation, Gaithersburg, MD, USA, 1993) Nucl. Instr. and Meth. A 347 (1994) 534.

[20] J.-C. Labiche and J.-P. Moy (preprint); J.-P. Moy and J. Morse, ref. [1] p. 265.

[21] M. Sutton, these Proceedings (8th Nat. Conf. on Synchrotron Radiation Instrumentation, Gaithersburg, MD, USA, 1993) Nucl. Instr. and Meth. A 347 (1994) 412.

[22] R. Pindak and S. Dierker (preprint). 